ANTIBIOTICS

\section{One-atom activity}

J. Am. Chem. Soc. 133, 13946-13949 (2011)

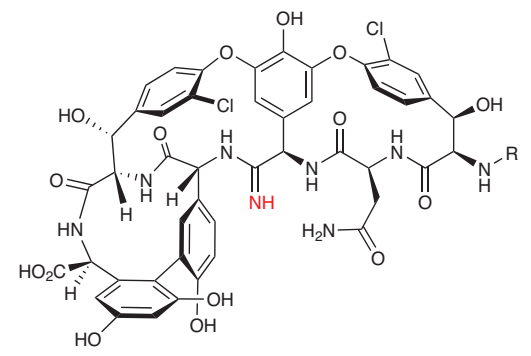

Vancomycin is used as the 'drug of last resort' in methicillin-resistant Staphylococcus aureus, and yet other bacteria are increasingly resistant to this glycopeptide antibiotic, posing a major problem for human health. Vancomycin activity results from binding to a D-Ala-D-Ala sequence within bacterial peptidoglycan; resistant bacteria use late-stage remodeling of this sequence to D-Ala-D-Lac-converting an amide bond to an ester bond- to circumvent vancomycin's effects. This single-atom change by the bacteria causes the loss of a hydrogen bond and the introduction of charge repulsion between this oxygen and the carbonyl group of residue 4 in the vancomycin scaffold. Crowley and Boger have previously shown that removal of the carbonyl somewhat allays these problems, improving both binding to the D-Ala-DLac sequence and activity against relevant resistant bacteria, but the overall change in activity was somewhat limited. Xie et al. now introduce a nitrogen to this same position to more directly compensate for the bacterial change. The careful conversion of a thiolated species to the amidine yielded a compound that almost fully restored binding and activity against resistant bacteria yet maintained a comparable level of activity against wild-type strains. These results provide a new strategy for antimicrobial drug discovery and add to our limited understanding of amidine as an amide isostere.

\section{TRANSLATION}

\section{Recoding worms}

J. Am. Chem. Soc., published online 8 August 2011, doi:10.1021/ja2054034

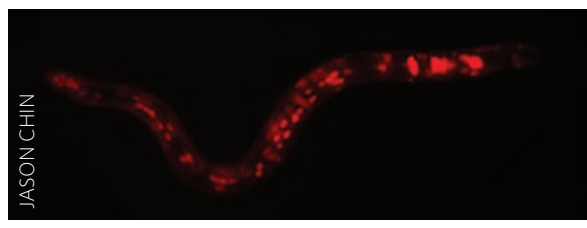

Reprogramming of translational stop codons to direct the incorporation of non-natural amino acids into proteins-or 'genetic code expansion', as it is sometimes called-has provided a useful method for the sitespecific engineering of proteins in bacteria, yeast and mammalian cells. These cellular approaches require an orthogonal coding system that makes use of an engineered mRNA, a suppressor tRNA and a matched aminoacyl-tRNA synthetase (AARS) that uniquely charges the suppressor tRNA with the non-natural amino acid of interest. Greiss and Chin now tackle the challenge of extending this technology to multicellular organisms by expanding the genetic code of the nematode Caenorhabditis elegans. Using a C. elegans strain in which the nonsensemediated decay (NMD) pathway had been disabled, the authors adapted an orthogonal

\section{NEUROSCIENCE}

\section{How to fill a vesicle}

The filling of synaptic vesicles with neurotransmitters such as glutamate is regulated at multiple levels and most importantly by the $\mathrm{H}^{+}$electrochemical driving force $\left(\Delta \mu_{\mathrm{H}_{+}}\right)$that is generated by the vacuolar $\mathrm{H}^{+}$-translocating ATPase (V-ATPase). $\Delta \mu_{H_{+}}$consists of a chemical gradient $(\Delta \mathrm{pH})$ and membrane potential $(\Delta \psi)$. When the anionic transmitter glutamate enters synaptic vesicles, it dissipates $\Delta \psi$, enabling the $\mathrm{H}^{+}$-ATPase to create $\Delta \mathrm{pH}$. However, vesicular glutamate transport is itself driven by $\Delta \psi$, and it has remained unclear whether $\Delta \psi$ might be regenerated from the accumulated $\Delta \mathrm{pH}$, enabling the vesicle to fill with greater amounts of the transmitter. From among the possible mechanisms to dissipate $\Delta \mathrm{pH}$, Goh et al. identified coupled cation $\left(\mathrm{Na}^{+}\right) / \mathrm{H}^{+}$exchange activity that could reduce the $\Delta \mathrm{pH}$ of synaptic vesicles but, because of the electroneutrality of the exchange, would maintain the $\Delta \psi$ produced by the $V$-ATPase activated by dissipation of $\Delta \mathrm{pH}$. Cations indeed stimulate either the uptake of glutamate into synaptic vesicles, an effect measured physiologically through an increase in quantal size, or the postsynaptic response to release of a single vesicle. Finally, the authors found that $\mathrm{Na}^{+}$/ $\mathrm{H}^{+}$exchangers (NHEs) mediate the stimulation of vesicular glutamate transport by cations. Overall, the results suggest that regulation of quantal size occurs via an increase in membrane potential mediated by $\mathrm{K}^{+} / \mathrm{H}^{+}$exchange.
tRNA-AARS system from the archaeobacter Methanosarcina mazei that had been previously optimized to encode nonnatural lysine analogs. An mRNA construct that produced green fluorescent protein (GFP) in the absence and mCherry in the presence of amber codon suppression by the orthogonal tRNA demonstrated that codon reprogramming takes place in the worm cells and requires the presence of the nonnatural amino acid. The incorporation of the modified lysine analogs was also verified directly by protein immunoblotting. The study provides the first example of genetic code expansion in an animal, representing a technology that enables more direct analysis of molecular events in living organisms. TLS

\section{SIGNALING}

\section{Clathrin pitstops}

Cell 146, 471-484 (2011)

Clathrin-mediated endocytosis (CME) regulates the uptake of proteins at the plasma membrane as well as the entry of pathogens and viruses and involves the formation of clathrin-stabilized endocytic structures. The $\mathrm{N}$-terminal $\beta$-propeller domain (TD) of the clathrin heavy chain is believed to be a central protein-protein interaction hub important for CME, but no tools interfering with clathrin have been available for studying the acute effects of disrupting TD-protein interactions. Now von Kleist et al. report two molecules named pitstop 1 and 2, which are based on distinct chemical scaffolds and inhibit association of clathrin TD with other endocytic proteins. In structures determined by X-ray crystallography, both molecules bind an interface between the first and second blades of the seven-bladed $\beta$-propeller, overlapping with the interface where clathrin-associating proteins bind. Application of pitstop 2 to cells blocked clathrin-mediated processes but did not affect clathrin-independent processes. Using total internal reflection fluorescence (TIRF) microscopy and fluorescence recovery after photobleaching (FRAP), the authors found that pitstop 2 perturbed clathrincoated pit dynamics but did not block pit assembly or the sequestration of cargo into clathrin-containing structures. These data unexpectedly indicate that TD-protein interactions are important for endocytic pit dynamics but not for clathrin assembly. Future applications of these molecules should help to shed light on the mechanisms that regulate the transitions from pit assembly to maturation and to disassembly. In addition, these molecules could serve as inhibitors of virus and pathogen entry. 